\title{
Le tramway, un objet industriel révélateur de l'évolution de la notion d'accessibilité aux personnes à mobilité réduite
}

\section{Changes in the concept of accessibility to people with reduced mobility as revealed by the tram}

\section{Laetitia Menétrieux a, Éric Heyrman b *}

\author{
Adresses professionnelles des auteurs à la date d'acceptation de l'article \\ a Service technique des remontées mécaniques et des transports guidés (STRMTG), 1461 rue \\ de la Piscine, 38400 Saint Martin d'Hères \\ b Institut National de Recherche sur les Transports et leur Sécurité (INRETS), Laboratoire \\ d'Ergonomie et de Sciences Cognitives pour les Transports (LESCOT), 25 avenue François \\ Mitterrand, Case 24, 69675 Bron cedex
}

Article reçu le 3 juillet 2006 ; accepté le 13 février 2007

courriel : eric.heyrman@,inrets.fr

\begin{abstract}
Résumé Cet article vise à expliciter la signification de la notion d'accessibilité et son évolution temporelle et à en révéler les facteurs explicatifs. Dans cette optique, deux approches, dites par le haut et par le bas, ont été utilisées. On s'interroge d'une part sur le rôle tenu par l'instrument juridique dans l'évolution de l'accessibilité et l'on s'appuie d'autre part sur l'étude de plusieurs projets d'implantation de tramway pour faire ressortir les facteurs qui ont favorisé l'émergence des différentes solutions techniques. Cette étude a révélé un niveau d'accessibilité en constante progression, selon une dynamique marquée par des processus d'accumulation et de reformulation de la signification de la notion d'accessibilité. Plusieurs facteurs peuvent expliquer cette évolution de signification, à savoir le travail en commun de cinq acteurs principaux, le développement d'une expertise technique sur l'accessibilité, des prises de position internationales et la création de la catégorie de personnes à mobilité réduite.
\end{abstract}

\begin{abstract}
This paper sets out to clarify the concept of accessibility and how it has changed over time. It will also attempt to identify the factors that explain these changes. With these goals in view two processes, a top-down and a bottom-up process, are considered. With regard to the first, we shall consider the role played by legal instruments in these changes in the concept of accessibility. With regard to the second, we shall examine several tram projects in order to highlight the factors which encouraged the emergence of various technical solutions. The study reveals that the level of accessibility has been improving continuously. This dynamic is marked by the accumulation of knowledge in the area and a reformulation of the concept of accessibility. Several factors can explain the changes in the concept: joint work on the part of five main actors, the development of technical expertise on accessibility, international stances and the creation of the category of people with reduced mobility.
\end{abstract}

Mots clés Accessibilité : Personnes handicapées ; Personnes à mobilité réduite ; Tramway ; Innovation 
Keywords Accessibility; People with disabilities; People with reduced mobility; Tram; Innovation

\section{Introduction}

L'une des principales améliorations des transports urbains de ces vingt dernières années est, d'après Claude Soulas (2003), leur accessibilité aux diverses catégories d'usagers et plus particulièrement aux personnes à mobilité réduite. Le tramway est un objet industriel qui illustre parfaitement cette tendance structurelle d'amélioration de la qualité d'usage. En effet, la lente maturation décisionnelle et technique des projets d'implantation des tramways a fréquemment facilité le développement d'un confort d'usage destiné à tous les usagers. Ainsi, depuis les premiers développements réalisés pour le tramway de Grenoble, son niveau d'accessibilité n'a cessé de croître. Nous proposons ici d'expliciter cette notion d'accessibilité aux personnes à mobilité réduite ainsi que son évolution temporelle et de révéler les facteurs qui ont facilité le changement de signification de la notion d'accessibilité. Cette contribution s'appuie sur nos activités de recherche, réalisées dans le cadre de travaux de fin d'études d'ingénieur (Heyrman, 2002), (Menétrieux, 2005a) et de masters universitaires (Heyrman, 2003), (Menétrieux, 2005b).

Afin d'expliquer le changement de sens de l'accessibilité et plus particulièrement du tramway accessible, nous avons exploré deux approches différentes: l'une dite par le haut, où l'innovation est impulsée par des acteurs nationaux et diffusée au niveau local; et une seconde approche, dite par le bas, où l'innovation est la conséquence d'interactions liant de multiples acteurs, porteurs d'intérêts hétérogènes et de conceptions éventuellement différentes des problèmes à résoudre (Meny et Thoenig, 1989). La recherche des dynamiques expliquant l'évolution de la notion d'accessibilité, qui prend chair dans chaque projet d'implantation de tramway, va nous permettre de déterminer le poids explicatif de chacune de ces deux approches, souvent présentées comme concurrentes. Afin de répondre à cette question, il est apparu pertinent d'examiner plusieurs projets d'implantation de tramway en France (Grenoble, Strasbourg, Lyon et Paris) afin de repérer leur niveau d'accessibilité et de mettre en lumière les facteurs qui ont facilité localement l'émergence de telle ou telle solution. À cet effet, les sources recueillies furent de nature diverse : ce sont autant des sources écrites (textes juridiques, archives administratives et documents techniques) que des sources orales (entretiens semi-directifs des acteurs locaux).

Nous présenterons dans une première partie l'instrument juridique utilisé par l'État français qui, s'il n'a explicité que récemment la notion d'accessibilité, n'en a pas moins créé un cadre institutionnel et cognitif contraignant pour les acteurs locaux. Nous montrerons dans une seconde partie comment la signification de tramway accessible aux personnes à mobilité réduite s'est peu à peu modifiée sous l'effet de volontés et d'actions locales, avant de revenir dans une dernière partie sur les facteurs qui expliquent ce changement de signification.

\section{L'outil juridique, entre contrainte symbolique et cadrage institutionnel}

\subsection{Une définition juridique très récente}

En 2005, la France a réformé sa politique en faveur des personnes handicapées en adoptant la loi n²005-102 du 11 février 2005 pour l'égalité des droits et des chances, la participation et la citoyenneté des personnes handicapées - concrétisation de la volonté du président de la 
République de faire de l'insertion des personnes handicapées dans la société l'un des trois chantiers prioritaires de son second quinquennat. Un vaste pan de cette loi concerne l'accessibilité à l'enseignement, à l'emploi, au cadre bâti et aux transports. Dans ce dernier domaine, elle impose une obligation de résultat: la mise en accessibilité des services de transport collectif dans un délai de dix ans. Elle introduit la notion de chaîne du déplacement, composée du cadre bâti, de la voirie, des aménagements des espaces publics, des systèmes de transport et de leur intermodalité. Cette chaîne du déplacement doit être organisée pour permettre son accessibilité dans sa totalité aux personnes handicapées ou à mobilité réduite. La loi n²005-102 impose d'une part aux autorités organisatrices des transports d'élaborer un schéma directeur d'accessibilité des services de transport collectif dans un délai de trois ans à compter de la publication de cette loi. D'autre part, les maires (ou les présidents de l'établissement public de coopération intercommunale si celui-ci a reçu cette compétence) doivent publier un plan de mise en accessibilité de la voirie et des espaces publics présents sur l'ensemble du territoire de leur commune. Si la loi n²005-102 impose une obligation de résultats et des instruments de politique publique, elle ne prévoit toutefois pas de délais, tant pour l'élaboration de ce plan que pour la mise en accessibilité de la voirie. Au-delà de ces outils de programmation, la loi $\mathrm{n}^{\circ} 2005-102$ comporte également des obligations de moyens. Ainsi, tout matériel roulant acquis pour renouveler les parcs de véhicules ou développer les réseaux de transport doit être accessible aux personnes handicapées et à mobilité réduite. Enfin, cette loi précise que l'octroi des aides publiques favorisant le développement des systèmes de transport collectif est subordonné à la prise en compte de l'accessibilité.

Un décret récemment publié est venu préciser ce qu'est un matériel roulant accessible : il s'agit du décret $n^{\circ} 2006-138$ du 9 février 2006 relatif à l'accessibilité du matériel roulant affecté aux services de transport public terrestre de voyageurs. Celui-ci désigne les bénéficiaires de l'accessibilité, en l'occurrence les personnes en situation de handicap et les personnes à mobilité réduite. Le premier public cible est défini par la loi n²005-102 : ainsi, constitue un handicap au sens de cette loi toute limitation d'activité ou restriction de participation à la vie de la société subie dans son environnement par une personne en raison d'une altération substantielle, durable ou définitive d'une ou plusieurs fonctions physiques, sensorielles, mentales, cognitives ou psychiques, d'un polyhandicap ou d'un trouble de santé invalidant. La notion de personnes à mobilité réduite (PMR) est reprise de celle présente dans la directive européenne $\mathrm{n}^{\circ} 2001 / 85 / \mathrm{CE}$ du 20 novembre 2001, à savoir toutes les personnes ayant des difficultés pour utiliser les transports publics, telles que, par exemple, personnes handicapées (y compris les personnes souffrant de handicaps sensoriels et intellectuels et les personnes en fauteuil roulant), personnes handicapées des membres, personnes de petite taille, personnes transportant des bagages lourds, personnes âgées, femmes enceintes, personnes ayant un caddie et personnes avec enfants (y compris enfants en poussette).

Le décret n²006-138 ne précise pas uniquement les bénéficiaires de l'accessibilité, il indique également les divers dispositions et aménagements s'imposant au matériel roulant — sans toutefois définir les exigences s'appliquant au système de transport dans sa continuité. Ainsi, la conception et les équipements du matériel roulant doivent permettre aux personnes en situation de handicap et aux personnes à mobilité réduite :

- d'effectuer les opérations de montée et de descente des véhicules ;

- de bénéficier de tous les services offerts à l'intérieur de la rame, sauf en cas d'impossibilité technique avérée qui donnera lieu à la mise en place de mesures de substitution ; 
- de se localiser, de s'orienter et de bénéficier en toute circonstance de l'information nécessaire à l'accomplissement du voyage.

Ces actions doivent être réalisées dans des conditions égales à celles des autres usagers, avec la plus grande autonomie possible et sans danger. Plus précisément, les aménagements propres à assurer l'accessibilité du matériel roulant doivent satisfaire aux obligations suivantes :

- s'il subsiste entre les seuils de porte et le quai des lacunes horizontales et/ou verticales non franchissables, celles-ci doivent être comblées grâce à l'ajout d'un équipement adéquat, à quai ou embarqué ;

- au moins une porte par rame permet le passage d'un fauteuil roulant ;

- les rames contiennent au moins un emplacement destiné aux personnes en fauteuil roulant et des sièges réservés aux passagers à mobilité réduite ;

- toute information délivrée à bord ou nécessaire au bon déroulement du voyage est diffusée sous forme sonore, visuelle et adaptée aux capacités de perception et de compréhension des personnes handicapées et à mobilité réduite.

Selon le décret $\mathrm{n}^{\circ} 2006-138$, les rames ferroviaires et de transport guidé faisant l'objet d'une modification substantielle doivent également être adaptées. De telles rames rénovées ne doivent respecter les exigences du décret $n^{\circ} 2006-138$ que si le marché public a été contracté plus d'un an après la publication d'un arrêté d'application. Cet arrêté, spécifique aux matériels guidés, précisera notoirement les dispositions techniques exigibles aux matériels roulants nouvellement acquis et celles exigibles aux matériels rénovés.

Ainsi l'accessibilité est clairement explicitée par les textes juridiques publiés depuis 2005. Les verbes employés définissent cette notion d'accessibilité : monter, descendre, accéder, s'orienter, se repérer, être informé. Trois expressions la qualifient : dans les mêmes conditions que les autres usagers, avec la plus grande autonomie possible et sans danger. Enfin ses bénéficiaires sont les personnes handicapées et les personnes à mobilité réduite.

\subsection{Une régulation juridique antérieure d'une autre nature : l'accessibilité entre objectif et moyen de politique publique}

Même si la loi n²005-102 a profondément réformé la politique française et si elle définit pour la première fois la notion d'accessibilité, la mobilité des personnes handicapées est un problème public inscrit de longue date sur l'agenda institutionnel de l'État français. Le 30 juin 1975 fut ainsi promulguée la loi nº75-534 d'orientation en faveur des personnes handicapées qui prônait l'accès aux institutions ouvertes à l'ensemble de la population chaque fois que les aptitudes des personnes handicapées et de leur milieu leur permettaient. Dans le domaine des transports, cet objectif de la loi n'était pas aussi explicite : il était renvoyé à des textes réglementaires d'application. Le décret $\mathrm{n}^{\circ} 78-1167$ du 9 décembre 1978 fixant les mesures destinées à rendre accessibles aux personnes handicapées à mobilité réduite les installations ouvertes au public existantes appartenant à certaines personnes publiques et à adapter les transports publics pour faciliter les déplacements des personnes handicapées explicita cet objectif : l'amélioration de la mobilité des personnes handicapées. Il imposa aux collectivités territoriales l'élaboration d'un programme d'aménagement des services et des 
installations de transports dans un délai de trois ans. Ces programmes devaient déterminer les mesures à mettre en œuvre pour :

- améliorer l'accessibilité des services réguliers de transport public aux personnes handicapées dans la mesure où le type du service et les contraintes d'exploitation de celui-ci ne sont pas manifestement incompatibles avec la nature même du handicap, ou

- mettre à la disposition des personnes handicapées un système de transport répondant à leurs besoins.

Si l'on reprend les travaux du groupe de travail interministériel chargé de recenser les problèmes posés par les transports des personnes handicapées, d'analyser les différentes solutions possibles et de préparer les textes réglementaires (Artaud-Macari et Bachelier, 1977), ces deux solutions correspondaient à deux populations distinctes: la mise en accessibilité pour les personnes légèrement handicapées ou autonomes et la création de services spécialisés pour les personnes lourdement handicapées ou non autonomes — dont les personnes en fauteuil roulant étaient, à l'époque, le stéréotype.

La politique nationale des années 1970-1990 privilégiait donc une approche très pragmatique : pour ne pas entraver les initiatives locales aucun texte réglementaire ne donna de prescriptions techniques en matière d'accessibilité des moyens de transport. De plus, la loi $\mathrm{n}^{\circ} 75-534$ n'avait pas donné de définition du handicap et donc a fortiori des personnes handicapées qui devaient bénéficier de l'accessibilité. Ainsi, comme l'avait précisé Mme Simone Veil lors des débats parlementaires (Chapireau, 1988a), (Chapireau, 1988b), est handicapée une personne déclarée comme telle par les commissions administratives compétentes localement (à savoir la commission départementale d'éducation spéciale pour les enfants et la commission technique d'orientation et de reclassement professionnel pour les adultes). Un objectif de politique publique était précisé : l'amélioration de la mobilité des personnes handicapées. Mais aucun moyen n'était privilégié — son choix étant à la discrétion des acteurs locaux, qui avaient la responsabilité de définir la notion d'accessibilité, et par voie de conséquence, les bénéficiaires de cette accessibilité.

Ainsi le niveau d'accessibilité que l'on peut actuellement observer sur les tramways modernes ne peut s'expliquer par une prescription impérative stricte de l'État. Jusqu'en 2005, celui-ci n'a pas utilisé d'outil réglementaire pour imposer une définition de l'accessibilité. Or, depuis sa réimplantation à Nantes, le tramway moderne n'a cessé d'accroître son accessibilité et son public cible. Nous nous proposons de montrer dans la suite de l'article que les solutions successivement retenues sont très largement liées aux acteurs locaux impliqués dans les projets, à leurs contraintes, leurs enjeux et leur vision de l'accessibilité. C'est pourquoi nous souhaitons examiner quelques projets d'implantation de tramway pour suivre cette lente maturation de la définition d'un tramway accessible - tout en nous gardant de fournir une approche naturaliste de cette évolution technologique, qui comme le suggère Bruno Latour (Latour, 1992) a été très probablement, à chaque étape, influencée par des enjeux forts et qui fut accompagnée de nombreux changements, notamment culturels.

\section{Un projet de tramway, une configuration d'acteurs, des solutions d'accessibilité}

Sur l'initiative du secrétaire d'État aux transports Marcel Cavaillé, un concours international d'idées a été lancé en 1975 portant sur l'établissement d'un projet définissant les 
caractéristiques d'un véhicule terrestre de transport de voyageurs, guidé, électrique, pouvant circuler sur la voirie banale et en site réservé et pouvant résoudre les problèmes de circulation des grandes agglomérations qui ne développent pas de système métropolitain. De ce concours d'idées est né le tramway moderne. Depuis le premier projet nantais, un vaste mouvement de réimplantation du tramway a eu lieu en France.

Nous n'examinerons pas chaque projet de tramway de la quinzaine d'agglomérations françaises qui ont déjà franchi ce pas (Fig. 1). Nous préférons nous concentrer sur quelques projets représentatifs d'une certaine conception de l'accessibilité, en nous focalisant sur le système d'acteurs qui a œuvré à améliorer l'accessibilité, ses contraintes-enjeux et ses représentations cognitives qui ont pesé sur les projets.

Fig. 1 Frise chronologique des réimplantations de tramways en France

\subsection{Grenoble, une ville initiatrice}

\subsubsection{L'intégration des personnes handicapées dans la société déjà inscrite à l'agenda politique}

Si le projet nantais de réimplantation du tramway aboutit dès 1985, la mise en accessibilité de ce mode de transport ne réussit à s'inscrire véritablement à l'agenda institutionnel des autorités locales qu'à Grenoble. En effet, cette ville était déjà préparée à une telle demande. Durant de nombreuses années, Grenoble était le seul endroit regroupant structure médicale et enseignement, avec un des campus universitaires les plus accessibles de France. Des actions expérimentales de mise en accessibilité du logement et de la voirie avaient été menées au cours des années 1970 en trustant une grande partie des subventions étatiques. La sensibilité des élus locaux aux problèmes des personnes handicapées s'est révélée en 1971 par l'élection au conseil municipal grenoblois d'une personne handicapée et en 1976 par le recrutement d'un architecte urbaniste spécialiste de cette question. En ce qui concerne la question de la mobilité des personnes handicapées, la ville de Grenoble y avait déjà répondu en partie en créant en 1979 un service spécialement adapté, résultat d'une initiative des associations de personnes handicapées visuelles et motrices. Cette création fut facilitée par la subvention accordée par la direction des transports terrestres (ministère des transports), qui combla le déficit d'exploitation de ce service pendant les premières années de son fonctionnement. Ainsi, avant le projet d'implantation du tramway, Grenoble répondait aux objectifs nationaux par la création d'un service spécifique, alors même que cette collectivité territoriale n'avait pas élaboré un programme d'aménagement de son système de transport collectif.

Lorsque le projet d'implantation d'un tramway devint mature, les associations de personnes handicapées se mobilisèrent et se manifestèrent auprès des acteurs locaux - essentiellement l'autorité organisatrice des transports et la mairie de Grenoble — pour revendiquer une mise en accessibilité de ce nouveau mode de transport. Une telle interpellation des autorités locales n'était pas saugrenue : depuis l'élection d'Hubert Dubedout à la mairie de Grenoble en 1965 un vaste mouvement de consultation et de participation des citoyens avait eu lieu. Ainsi à cette époque, l'urbanisme était l'affaire de tous (Ratel, 1996). La question de l'accessibilité fut traitée de la même manière. Un collectif d'associations de personnes handicapées moteurs, regroupées autour de l'Association des Paralysés de France, fut constitué. Des contacts formels entre ce collectif et les autorités publiques, et d'autres contacts plus personnels (Collomb d'Eyrames, 2002), se nouèrent. Le collectif d'associations fit pression, à de 
nombreuses reprises, sur la mairie de Grenoble et sur l'autorité organisatrice des transports, afin de participer aux études de définition d'un matériel roulant accessible.

Toutefois, malgré la bonne volonté des élus locaux, des contraintes techniques persistaient. En effet, le matériel qui devait être implanté à Grenoble était celui issu du concours Cavaillé, construit par Alsthom, - matériel qui ne prenait pas en considération les besoins des personnes handicapées. Les élus grenoblois interpellèrent le ministère des transports, en particulier une instance qu'il venait de mettre en place, pour confronter les demandes des collectivités locales aux contraintes du groupement d'industriels du concours Cavaillé, ceci afin de rendre compétitif le produit français à l'exportation : le comité technique tramway français standard.

\subsubsection{Le comité technique tramway français standard comme structure nationale d'appui}

Cette instance regroupait notamment la direction des transports terrestres, Alsthom, plusieurs autorités organisatrices des transports (en l'occurrence celles de Grenoble, Nantes, Strasbourg, Saint-Étienne, Lille, Marseille, Toulouse, ainsi que le groupement des autorités responsables des transports), leurs sociétés exploitantes, les maîtres d'œuvre (Semaly) et des centres d'expertise (CETUR et IRT). La première réunion de ce comité fut consacrée au problème de l'accessibilité. Une étude du CETUR y fut présentée. Trois solutions étaient possibles, aucune n'étant exempte d'inconvénients : une plate-forme élévatrice à quai ou embarquée, des quais hauts ou un plancher du véhicule plus bas. La première possibilité fut rejetée pour des raisons d'intégration architecturale et de perte de temps d'exploitation. La seconde solution semblait impossible à Grenoble et Strasbourg, une nouvelle fois pour des questions d'inscription urbanistique. La dernière possibilité nécessitait une remise en cause totale du véhicule. À l'issue de cette réunion, il fut décidé d'évaluer les incidences financières de chaque solution.

\subsubsection{Une action volontariste des acteurs locaux}

Toujours incités à agir par les associations locales de personnes handicapées, les élus grenoblois se sont appuyés sur l'expertise de l'entreprise qui exploite son réseau de transport, celle de la maitrise d'œuvre et celle du designer industriel en charge de la conception du véhicule pour convaincre Alsthom de modifier substantiellement le projet initial. En effet, Alsthom, qui avait à l'époque une culture industrielle marquée par ses relations étroites avec la SNCF, n'avait pas encore pris conscience de la nécessité de répondre aux demandes diversifiées des collectivités locales. Or la SNCF ne demandait aucune innovation en matière d'accessibilité aux personnes handicapées. De plus, celle-ci précisait strictement sa demande et Alsthom réalisait scrupuleusement la prestation selon un cahier des charges très descriptif, alors que celui du tramway de Grenoble - mais également ceux du métro de Lyon et du tramway de Nantes - étaient beaucoup plus fonctionnels. Ces éléments expliquent que l'initiative de déplacer les équipements de traction du tramway français standard initialement installés dans le plancher du véhicule vers le plafond (Fig. 2) ne soit pas venue des ingénieurs d'Alsthom. Elle est issue de la confrontation des idées et propositions des différents membres du comité technique tramway français standard.

\section{Fig. 2 Principes d'aménagement du tramway grenoblois}


Ainsi, alors que la situation semblait bloquée en raison des contraintes techniques (notamment $\mathrm{du}$ fait de l'encombrement du moteur) et des préoccupations de bonne inscription urbanistique, la troisième solution envisagée devint réalisable avec la perspective d'abaisser le plancher du véhicule. Cette solution était devenue crédible techniquement dès 1984 avec la mise en exploitation d'un tramway à plancher mi-bas par l'agglomération de Genève, Une réunion fut organisée pour lever les derniers obstacles à un tel projet. Le président de l'autorité organisatrice des transports grenoblois demanda l'appui du ministre des transports, qui était sensibilisé à la question de l'accessibilité des personnes handicapées. En effet, le ministre Fiterman adressa aux préfets un rappel à l'ordre, via une lettre-circulaire datée du 10 mai 1982, leur indiquant que les programmes d'aménagement du système de transport collectif prévus par le décret $\mathrm{n}^{\circ} 78-1167$ devaient être élaborés. Le ministre fut représenté par son directeur de cabinet adjoint. Au cours de cette réunion, il est apparu que les difficultés techniques cachaient des préoccupations financières : la mise en accessibilité ne va-t-elle pas accroître le coût du véhicule et faire diminuer son attractivité à l'exportation ? Les différentes parties se sont mises d'accord pour un partage du coût du développement industriel : le ministère des transports décida de prendre en charge $50 \%$ de la dépense, Alsthom accepta d'en prendre $30 \%$ pour des raisons stratégiques, $20 \%$ restant à la charge de l'agglomération grenobloise. Pour dédommager cette dernière du coût de cette recherche et développement supplémentaire, un mécanisme de royalties fut mis en place, applicable dès lors que d'autres agglomérations souhaitaient utiliser la technologie développée.

La RATP, présente à cette réunion, s'était engagée, quant à elle, à réaliser gratuitement le développement d'une palette automatique si cette dernière s'avérait nécessaire. L'autorité organisatrice des transports mandata l'Institut de recherche sur les transports (IRT) pour étudier si, avec un tel système de plancher bas, les personnes en fauteuil roulant pouvaient accéder sans palette. Des essais sur maquette montrèrent que les lacunes verticales et horizontales restaient trop élevées pour être franchies sans danger: une palette était indispensable (Florès et al., 1984).

\subsubsection{Des solutions d'accessibilité centrées sur le handicap moteur}

La mobilisation de tous ces acteurs a abouti à la conception d'un véhicule à plancher bas sur les deux tiers de sa longueur: seules les deux extrémités sont surélevées de trois marches (pour y implanter les bogies moteurs). Deux palettes équipent chaque rame (cf. photo 1), alors que chaque véhicule comporte quatre portes. Le déploiement de la palette était initialement actionné à l'aide d'un bouton installé sur le quai de la station de tramway, mais ce système fut rapidement abandonné, car trop nombreuses étaient les personnes qui actionnaient ce dispositif indûment. Le conducteur fut alors chargé de déployer la palette, en entrée, dès qu'il apercevait une personne en fauteuil roulant sur le quai, en sortie, lorsque la personne handicapée actionnait un bouton situé à côté de l'emplacement réservé.

\section{Photo 1 Palette du tramway de Grenoble déployée, modèle de 1987}

À noter que, reflétant le type des associations qui se sont mobilisées, les solutions d'accessibilité répondent principalement au problème du handicap moteur.

\subsubsection{L'expérience de Grenoble : un exemple suivi ?}

Après le développement technologique grenoblois, les tramways implantés en France ne pouvaient être qu'à plancher bas. En effet, il s'est avéré a posteriori que la mise en 
accessibilité du tramway permettait un gain de temps substantiel lors des montées et descentes de tous les usagers : il a ainsi été nécessaire de refaire toutes les fiches horaires du tramway de Grenoble pour intégrer ce gain de temps inattendu. En outre, les villes fondatrices qui ont implanté un tramway sur leur territoire cherchèrent à prouver la pertinence de ce projet et à changer l'image dépassée qu'avait le tramway auprès de leurs citoyens : la réussite grenobloise fut ainsi très largement médiatisée par de nombreux partisans des transports publics et par la municipalité grenobloise elle-même. Le comité technique tramway français standard fut également le lieu d'une sensibilisation des collectivités locales qui avaient un projet d'implantation de tramway. Enfin il existe une circulation de personnel entre les autorités organisatrices des transports ou les sociétés exploitantes des réseaux qui explique que l'expertise accumulée lors du projet grenoblois a été réutilisée pour les autres projets. Il en est ainsi pour le tramway de Strasbourg, où le designer industriel et l'entreprise en charge de la maîtrise d'œuvre avaient déjà travaillé sur le projet grenoblois.

\subsection{L'importation de bonnes pratiques : le projet de tramway de Strasbourg}

Candidate à la réimplantation d'un tramway et membre à ce titre du comité technique tramway français standard, l'agglomération de Strasbourg a longtemps hésité, dans les années 1980, entre un tramway et un métro automatique léger de type VAL. À la suite des élections municipales de 1989, l'option tramway fut retenue. Le matériel implanté à Strasbourg marqua une seconde étape dans l'évolution technologique : au début des années 1990, cette ville acheta au constructeur ABB un tramway à plancher bas intégral. Cette demande n'est pas venue, comme on aurait pu s'y attendre, des associations de personnes handicapées, car celles-ci étaient globalement satisfaites de la solution grenobloise. Ce sont des contraintes et des objectifs d'exploitation qui expliquent cette demande. En effet, l'exploitant strasbourgeois a pu observer que les usagers ne s'installaient pas spontanément dans les parties surélevées aux deux extrémités du tramway grenoblois : aux heures de pointe, ils craignaient de ne pas pouvoir sortir à temps de la rame. Après avoir visité plusieurs réseaux européens, les élus, le maître d'œuvre et les techniciens de l'autorité organisatrice des transports strasbourgeoise étaient convaincus que la solution grenobloise était dépassée : un plancher intégralement plat leur semblait favorable pour faciliter les correspondances tram-bus et l'intégration urbanistique du tramway dans la ville - ce mode de transport étant vu comme un trottoir roulant. Cette solution était devenue réaliste depuis que deux constructeurs italiens avaient développé, chacun de leur côté, un nouveau bogie moteur. De plus Brême avait inauguré en 1990 un tramway à plancher bas intégral dont la particularité est de disposer ses moteurs sous les sièges des usagers. Un appel d'offres fut lancé sur la base d'un cahier des charges d'un tramway à plancher surbaissé sur $100 \%$ de sa longueur, l'accessibilité aux personnes se déplaçant en fauteuil roulant étant complétée par deux palettes, de type grenoblois, installées sur les portes centrales de chaque rame.

Le tramway strasbourgeois est non seulement à plancher bas intégral sur toute la surface du véhicule (ceci grâce à de petits moteurs refroidis par eau), mais il présente surtout une innovation importante en matière d'accessibilité : des dispositifs d'annonces sonores et visuelles des stations dans les rames de tramway. La direction et le temps d'attente du prochain tramway sont en outre affichés dans la station. À Strasbourg, le problème de l'information des voyageurs a été inscrit à l'agenda politique des décideurs dès la conception du tramway. Ces différents développements technologiques matérialisent de manière concrète l'intégration des personnes déficientes auditives et visuelles dans les bénéficiaires de l'accessibilité. Le tramway grenoblois avait certes une annonce sonore des stations, mais le problème de mobilité des personnes aveugles et malvoyantes n'est vraiment apparu qu'avec 
l'inauguration de la deuxième ligne du tramway grenoblois en 1990, laquelle présentait un tronc commun avec la première : sans information adaptée, les personnes déficientes visuelles ne savaient pas quelle direction prenait le tramway en approche.

Il est toutefois frappant de constater que le projet strasbourgeois est, comparativement à Grenoble, marqué par l'absence de l'acteur étatique. La définition du niveau d'accessibilité a été réalisée par l'autorité organisatrice des transports et le maître d'œuvre après étude des expériences étrangères, notamment allemandes, et consultation des associations de personnes handicapées pour appréhender leurs besoins. L'instauration d'une relation de confiance entre techniciens et personnes handicapées, ainsi que la compréhension de leurs contraintes et enjeux respectifs semblent un impératif pour réussir un tel projet. À Strasbourg, les contacts avaient débuté lors de l'élaboration d'une charte Ville et handicap, finalement adoptée le 29 juin 1990 et se sont poursuivis avec la création d'un groupe de travail spécifique au projet d'implantation du tramway.

\subsection{Une remise en cause de l'accessibilité grenobloise : Lyon et Paris}

La définition de l'accessibilité a poursuivi son évolution : si dans les années 1980, l'accès au véhicule en toute sécurité et en toute autonomie était le problème principal, le caractère non discriminatoire de cet accès est désormais exigé. Les associations de personnes handicapées reprochent depuis peu au système de palette de Grenoble le fait de devoir se déclarer, puisque, à la montée, le conducteur doit reconnaître la personne handicapée et, à la descente, celle-ci doit appuyer sur un bouton spécifique. Elles regrettent en outre que deux portes sur quatre seulement soient équipées. Ainsi, alors que le système grenoblois de palette était perçu à l'origine comme une avancée, car évitant le dispositif technologique de l'élévateur ressenti comme ségrégatif, depuis la toute fin des années 1990, il est vu comme un système soumis à l'appréciation discrétionnaire du conducteur. Cette préoccupation nouvelle a été intégrée et prise en compte dans le projet de tramway de Lyon, pour lequel le comble-lacune, qui équipe chaque porte se déploie systématiquement à chaque ouverture, ou dans celui du tramway circulant sur les boulevards des Maréchaux (Paris), où le même résultat est obtenu à l'aide d'un seuil rétractable (Fig. 3).

Sans nous attarder sur le projet parisien qui vient d'être inauguré en décembre 2006, nous proposons d'examiner plus longuement le projet de Lyon.

\section{Fig. 3 Schéma de principe d'un tramway avec seuil rétractable non motorisé}

\subsubsection{L'accessibilité au transport a déjà une longue histoire tumultueuse à Lyon}

Dès 1973, à chaque construction d'une ligne de transport en commun en site propre, les associations de personnes handicapées ont revendiqué sa mise en accessibilité, mais de nombreuses contraintes (financières, d'exploitation, de sécurité, temporelles notamment) n'ont pas permis d'y parvenir pour les deux premières lignes de métro inaugurées en 1978. De ces actions résulta cependant la décision de prévoir des réserves de génie civil pour installer ultérieurement des ascenseurs dans les stations des prolongements de ligne. En 1991, la ligne D fut inaugurée avec l'intégralité de ses stations équipées en ascenseurs. En outre, un travail particulier fut réalisé pour assurer une bonne liaison entre le quai et la rame de cette ligne : à l'une des portes de chaque côté de la rame, un comble-lacune vint réduire les espaces horizontaux et verticaux. Un espace multi-usages fut créé dans la rame de métro pour les personnes en fauteuil roulant. 
En 1997, un plan de déplacements urbains fut adopté : il prévoyait que le réseau de transport collectif devait être rendu progressivement accessible aux personnes handicapées et à mobilité réduite. De cette décision découla la volonté de développer le service spécialement adapté aux personnes handicapées créé en 1980 et de lancer un programme décennal d'équipement des stations de métro en ascenseurs. Les deux lignes de tramway, principales mesures de ce plan de déplacements urbains, devaient être à l'image de cette volonté politique : accessibles à tous.

\subsubsection{Un chargé de mission à l'articulation des réseaux politiques et associatifs}

Afin d'impulser une certaine cohérence dans la politique d'accessibilité de la municipalité, le premier adjoint au maire en charge des transports, également président de l'autorité organisatrice des transports urbains et vice-président de la communauté urbaine de Lyon en charge des transports, a missionné l'un de ses anciens colistiers qui connaissait bien les besoins des personnes handicapées, étant lui-même utilisateur de fauteuil roulant et ayant milité au sein de plusieurs associations. Étant de plus le représentant du maître d'ouvrage auprès du constructeur pour les questions d'accessibilité, il assura donc un rôle de traduction des demandes des associations et des contraintes du constructeur auprès du maître d'ouvrage. Mais il était également porteur d'une définition de l'accessibilité qui lui était propre : celle d'un accès à tous, en toute autonomie et non discriminatoire, c'est-à-dire sans que la personne handicapée ait besoin de se signaler comme telle.

Le cahier des charges, établi par le maître d'œuvre, prévoyait un tramway à plancher bas. Une option permettait de présenter un projet à plancher bas intégral. La proposition retenue, celle d'Alstom, qui avait récemment développé sa gamme Citadis, intégra cette option. Le niveau d'accessibilité de ce tramway fut vérifié lors d'une réunion. Le constructeur, le chargé de mission accessibilité, l'autorité organisatrice des transports, son maître d'œuvre, l'exploitant, la direction départementale de l'équipement et les associations de personnes handicapées, essentiellement celles du handicap moteur, purent tester sur une maquette en bois l'interface quai-rame. Celle-ci se révéla non satisfaisante : les lacunes dépassaient $4-5 \mathrm{~cm}$. Elles étaient certes inférieures aux recommandations du COLITRAH ${ }^{1}$ (Fig. 4 et 5), mais les roues de certains fauteuils roulants se bloquaient.

\section{Fig. 4 Recommandations du Colitrah}

\section{Fig. 5 Dimensions d'un fauteuil roulant définies par la norme ISO 7193}

Avec l'appui du maire de Lyon et du président de l'autorité organisatrice des transports, le chargé de mission imposa au constructeur de modifier son dispositif. Quelques mois plus tard, il fut invité à tester le nouveau dispositif, un comble-lacune, à l'usine Alstom d'Aytré. La seconde réunion avec les associations confirma son avis : les lacunes de $25 \mathrm{~mm}$ ainsi obtenues étaient satisfaisantes pour le passage des personnes en fauteuil roulant. Ces personnes pouvaient en outre accéder au quai rehaussé de la station de tramway depuis la voirie environnante, par une rampe, au moins sur l'un des deux côtés.

Les personnes déficientes visuelles et auditives ne furent pas oubliées. Connaissant moins leurs problèmes de mobilité, le chargé de mission de la ville de Lyon a organisé des réunions

\footnotetext{
${ }^{1}$ Recommandations du Colitrah de mars 1998 précisées dans le document «Prescriptions et recommandations à tous des réseaux tramways »
} 
avec les principaux acteurs associatifs. Il fut décidé d'installer un système d'annonces sonores et visuelles des prochaines stations dans les rames de tramway, des vitres antireflets et un contraste de couleurs pour faciliter la lecture de la signalétique et de l'information de ligne, des bandes podotactiles sur les quais et les carrefours et des dispositifs sonores aux feux avoisinant le tramway.

Seul regret exprimé par le chargé de mission de la ville de Lyon : l'oubli des personnes handicapées mentales. Pour faciliter le déplacement de ces personnes qui ont des difficultés de compréhension de l'espace, il aurait été possible de différencier chaque station et lui donner une ambiance différente, à l'instar des stations de métro marseillaises. Il est étonnant de constater la non-prise en compte, au niveau local, des besoins des personnes handicapées mentales, alors même qu'ils commencent à être connus dès le milieu des années 1980, après que leurs associations nationales ont présenté leurs problèmes d'accès au sein du comité de liaison pour l'accessibilité des transports et lancé une étude afférente à cette question. Ces premiers travaux (Hermelin et al., 1986) et ceux qui ont suivi (Sanchez, 1997), (Sanchez et Velche, 1999) ont porté sur la signalétique et les capacités des personnes handicapées de se repérer. Des informations étaient donc à la disposition des acteurs locaux. Plusieurs solutions étaient envisageables : une amélioration de la signalétique, une formation à l'utilisation du service public de transport et la création d'un service d'accompagnement des personnes handicapées mentales dans leurs déplacements. Pourtant aucune de ces mesures ne fut mise en œuvre, en partie du fait de l'absence d'un acteur local, porte-parole des besoins des personnes handicapées mentales en matière de déplacements. En effet, autant leurs associations nationales étaient porteuses de revendications à ce sujet, autant leurs associations locales ne se sentaient pas directement concernées par le problème de l'accessibilité.

Le cas lyonnais se singularise des projets grenoblois et strasbourgeois par la présence d'un chargé de mission accessibilité qui fut porteur d'une vision de l'accessibilité — vision qui était en phase avec les demandes des associations et qui a trouvé une traduction concrète dans le matériel roulant.

\subsubsection{Le contrôle de l'État}

L'État a vérifié la mise en accessibilité du tramway lyonnais. En effet, une commission dite locale de sécurité avait été créée par arrêté préfectoral pour apporter aide et assistance aux services de contrôle de l'Etat qui ont la charge de proposer au préfet l'autorisation de mise en exploitation du système tramway. Elle était composée, entre autres, de la DDE du Rhône, des services de protection civile, d'incendie et de secours, du CERTU, de la communauté urbaine de Lyon et des communes concernées. Le correspondant accessibilité de la DDE du Rhône fut invité à y siéger. L'autorité organisatrice des transports, accompagnée de ses maîtres d'œuvre, venait exposer ses contraintes et difficultés. En ce qui concerne l'accessibilité, domaine dans lequel il y avait à l'époque un vide juridique, cette commission conseilla vivement à l'autorité organisatrice des transports d'utiliser, pour adapter ses quais de tramway, les normes réglementaires en vigueur dans les établissements recevant du public et de veiller à inclure les personnes malvoyantes et malentendantes - autant de prescriptions définitivement adoptées par l'autorité organisatrice des transports. D'un point de vue encore plus réglementaire, la commission consultative départementale de sécurité et d'accessibilité statua sur l'accessibilité de la station de tramway Perrache, car cette station faisait partie de la gare SNCF éponyme où s'appliquait la réglementation relative aux établissements recevant du public. 
Ainsi l'Etat change de posture : il n'est plus simplement financeur, via des subventions sur le matériel roulant ou des aides à la recherche et développement comme ce fut le cas à Grenoble. Il peut également s'inscrire dans une activité de contrôle, par l'intermédiaire de plusieurs outils mis à sa disposition.

\section{L'accessibilité, une innovation à la croisée de dynamiques individuelles et structurelles}

La présentation de ces différents projets d'implantation de tramway révèle un niveau d'accessibilité en constante progression - dynamique marquée par des processus d'accumulation et de reformulation de la signification de la notion d'accessibilité. Plusieurs facteurs peuvent expliquer cette évolution de signification : le travail en commun de cinq acteurs principaux, le développement d'une expertise technique sur l'accessibilité, des prises de position internationales et la création de la catégorie des personnes à mobilité réduite.

\subsection{Un quintette indispensable}

Cinq acteurs principaux ont œuvré sur chacun des projets examinés pour obtenir un tramway de plus en plus accessible : l'autorité organisatrice des transports, le maître d'œuvre, le constructeur de tramways, les associations de personnes handicapées et l'État. En dépit de contraintes et de d'enjeux différents, ces cinq acteurs ont réalisé un travail en commun fructueux aboutissant à la définition du tramway accessible.

Les autorités organisatrices des transports ont su mobiliser les énergies et les expertises et apporter les financements cohérents avec leur politique. Ainsi à Grenoble, le syndicat mixte des transports en commun a convaincu le constructeur de modifier son matériel. Il a mis tout son poids de client pour légitimer le fait de procéder à une nouvelle recherche et développement, alors qu'Alsthom travaillait encore sur le tramway français standard. Les autorités organisatrices des transports ont également joué un rôle de légitimation-filtrage des demandes des associations locales et de synthèse entre ces demandes et les contraintes des industriels.

Le maître d'œuvre joue véritablement un rôle de marginal sécant, c'est-à-dire qu'il est partie prenante dans plusieurs systèmes d'action en relation les uns avec les autres et qui peut, de ce fait, jouer le rôle indispensable d'intermédiaire et d'interprète entre des logiques d'action différentes, voire contradictoires (Crozier et Friedberg, 1977). En effet, il assure la traduction des demandes de l'autorité organisatrice des transports auprès du constructeur et celle des contraintes du constructeur auprès de son client. Son expertise, développée tout au long de ces projets d'implantation de tramway, permet de déterminer le niveau de prestation acceptable des points de vue technique, budgétaire ou d'exploitation.

Disposant d'une expertise technique très rare, les constructeurs occupent une position incontournable, mais ils sont également contraints par leur stratégie et leur culture d'entreprise. Nous avons déjà exposé les réticences d'Alsthom à intégrer les besoins des personnes handicapées lors du développement du tramway français standard. Principalement implantée dans le ferroviaire lourd avec comme client privilégié la SNCF qui au début des années 1980 n'exprimait pas de demandes particulières en matière d'accessibilité, cette entreprise a dû trouver bien incongrues les exigences de l'autorité organisatrice des transports grenobloise. Alsthom était en outre plutôt habitué à des discussions entre techniciens et son introduction dans le marché des tramways urbains a donc nécessité une acculturation. Il est 
toutefois à noter que, une fois convaincue, cette entreprise a montré une forte capacité d'adaptation et de réponse aux attentes des autorités organisatrices des transports.

Les associations de personnes handicapées ont joué le rôle d'aiguillon. Le tramway de Grenoble n'aurait jamais été accessible si les associations locales n'avaient interpellé les pouvoirs publics. Leur insistance a été un facteur de légitimation de l'action des autorités organisatrices des transports. Toutefois, toutes les associations ne se sont pas mobilisées suivant la même temporalité : les associations du handicap moteur, surtout celles regroupant des personnes circulant en fauteuil roulant, ont d'abord été porteuses de revendications, dès les années 1980. Contre toute attente, alors que la politique nationale privilégiait l'option des transports spécialisés pour les personnes en fauteuil roulant, ce furent les premières à revendiquer une accessibilité des systèmes conventionnels de transport collectif. Les demandes des personnes aveugles et malvoyantes, pourtant considérées au niveau national comme autonomes et donc bénéficiaires légitimes de l'accessibilité, n'ont émergé qu'au début des années 1990. Les associations des personnes déficientes auditives, marquées par une grande fragmentation, ont été consultées lors du projet lyonnais. Les associations des handicaps cognitifs et psychiques se signalent par leur absence au niveau local. Enfin, les associations de personnes handicapées disposaient d'une ressource rare : elles ont longtemps détenu le monopole de la définition des besoins des personnes handicapées.

L'État, tant central que local, a lui aussi contribué à la mise en accessibilité du tramway. Il a notamment légitimé les demandes de l'autorité organisatrice des transports grenobloise en participant financièrement au développement industriel du tramway français standard à plancher bas et en apportant son appui institutionnel. Il a ensuite participé à la diffusion du tramway accessible en conditionnant, depuis 2001, sa participation financière à l'achat d'un matériel roulant à son caractère accessible. Si l'État a facilité le développement industriel de l'objet tramway, il a récemment adopté une position plus régalienne de contrôle, comme l'a montré l'exemple lyonnais. Enfin l'État vient d'utiliser son pouvoir de contrainte normative en donnant pour la première fois une définition juridique de l'accessibilité.

\subsection{Développement d'une expertise technique}

L'évolution de la notion d'accessibilité s'explique également par le développement d'une expertise technique dans ce domaine. Plusieurs dynamiques ont contribué à ce développement : une circulation de techniciens au sein des différentes autorités organisatrices des transports ou de leurs exploitants, la participation de plusieurs acteurs aux différents projets, un double mouvement d'acculturation et de sensibilisation à travers des groupes de travail et une veille technologique réalisée par certains acteurs. Ainsi, il est frappant de constater que certains ingénieurs et techniciens ont travaillé sur plusieurs projets étudiés : il s'agit notamment de l'entreprise maître d'œuvre, du designer industriel et de plusieurs ingénieurs qui ont changé d'employeurs dans le cadre d'une évolution de carrière. Toutes ces personnes ont apporté leur expertise, ainsi que les retours d'expérience qu'elles ont pu tirer de projets antérieurs. Une telle mobilité explique largement pourquoi de nombreuses solutions d'accessibilité (mise en place de palettes, présence de marches aux extrémités du tramway) sont définies par opposition à ce qui existait précédemment. Un autre facteur réside dans l'existence de lieux d'échange : le premier fut le comité technique tramway français standard déjà évoqué. Une autre occasion fut les travaux réalisés dans le cadre de la mission dirigée par Madame la députée Fraysse-Cazalis (Florès, 1984), chargée d'une mission d'étude des solutions aux problèmes de l'accessibilité des transports et de la ville aux personnes handicapées. Le dernier lieu d'acculturation et de sensibilisation était le Comité de liaison 
pour l'accessibilité des transports (COLITRAH), qui regroupe l'État, les industriels, les autorités organisatrices des transports et les associations de personnes handicapées. Enfin, si le développement d'une expertise technique dans le domaine sur l'accessibilité a été facilité par la circulation de techniciens au sein des différentes entreprises de transport et l'existence de lieux d'acculturation et de sensibilisation, il peut également s'expliquer par la veille technologique réalisée de manière systématique par la maîtrise d'œuvre et les constructeurs de tramway et par l'analyse comparative des différents produits présents dans cette niche commerciale.

\subsection{L'accessibilité, une demande internationale}

Des prises de position d'instances internationales et européennes sont également venues appuyer la revendication d'une mise en accessibilité des transports en commun. Ainsi, l'ONU adopta en 1982 le Programme mondial d'actions sur les personnes handicapées et en 1993 les Règles pour l'égalisation des chances des personnes handicapées — dont la règle 5 est spécifique à l'accessibilité. La Conférence européenne des ministres des transports créa dès 1985 un groupe de travail sur l'accessibilité et l'inclusion. Ce groupe produisit de nombreux rapports et des recommandations sur les mesures à mettre en œuvre pour instaurer des conditions de transports sans obstacle (dont une Charte sur l'accès aux services et infrastructures de transports et un Guide de bonnes pratiques pour l'amélioration des transports pour les personnes à mobilité réduite en 1999). Un soutien politique de ce travail s'est exprimé à travers l'adoption de plusieurs résolutions du Conseil des ministres des transports. Enfin le principe de non-discrimination a été introduit dans le traité d'Amsterdam (1997) de la Communauté économique européenne.

Ces prises de position sont concomitantes d'un changement de définition internationale du handicap. Ainsi au début des années 1980, l'Organisation mondiale de la santé (OMS) a publié une classification internationale du handicap, fondée sur une vision médicale du handicap. Ce modèle conceptuel est centré sur la personne handicapée et ses limitations fonctionnelles (Ravaud, 1999). Le handicap repose sur une anomalie : l'écart entre les capacités de la personne handicapée et les capacités moyennes des individus valides. Les solutions techniques préconisées résidaient dans la création de services de transport spécialisés, adaptés aux besoins des personnes handicapées. Au cours de la décennie 1990, cette classification fut critiquée pour son caractère excessif. Récemment, l'OMS a adopté la classification internationale $\mathrm{du}$ fonctionnement qui réalise une synthèse entre les modèles médicaux et sociaux du handicap. Ce dernier modèle renverse le poids de la charge. Une personne n'est handicapée que dans une situation donnée : le regard doit donc être déplacé de la personne vers les situations handicapantes. L'action publique sous-jacente est alors l'adaptation du cadre de vie. Un tel renversement de la représentation internationale du handicap légitime des mesures favorisant l'accessibilité.

\subsection{Les Personnes à mobilité réduite, une catégorie incantatoire, mais non opératoire}

Les entrepreneurs politiques de l'accessibilité ont réussi à faire accepter l'idée que cette action publique ne concernait pas uniquement les personnes en fauteuil roulant mais qu'elle améliorait la situation de tous les usagers, l'attractivité des systèmes de transport collectif et leur degré de confort. Dès les années 1970, ils ont cherché à rapprocher les préoccupations des personnes handicapées avec celles des personnes vieillissantes — à un moment où l'espérance de vie des Français ne cessait d'augmenter. Afin de regrouper l'ensemble des bénéficiaires de l'accessibilité, ils ont inventé la notion de personnes à mobilité réduite. Selon 
les définitions données par les différents organismes (CEMT, Union européenne, CERTU, délégation ministérielle à l'accessibilité du ministère de l'équipement), cette catégorie rassemble les personnes traditionnellement reconnues comme handicapées (c'est-à-dire une personne qui présente un handicap physique, visuel, auditif, cognitif ou psychique), les personnes âgées, les femmes enceintes, les mères de famille (catégorie étendue récemment aux parents) avec poussette, les personnes avec des caddies ou des bagages, les personnes revenant accidentées du ski désignées comme handicapées temporaires, les étrangers, les illettrés, les personnes allergiques. La catégorie de personnes à mobilité réduite regroupe ainsi de nombreuses populations. Elle a l'avantage d'avoir un pouvoir très évocateur : qui peut avoir la certitude de ne jamais avoir à se considérer comme une PMR à un moment ou un autre de sa existence? Deux récentes études ont conforté l'argumentaire des promoteurs de l'accessibilité : selon l'Institut d'aménagement et d'urbanisme de la région d'Île-de-France $35 \%$ des personnes ont des difficultés de déplacement (IAURIF, 1997) ; 40,4\% des personnes déclarent une déficience d'après une étude de l'INSEE (Hauet et Ravaud, 2002). Or depuis les lois de décentralisation, les élus locaux ont bâti la légitimité de leurs institutions et de leurs mandats sur leur capacité de résoudre les problèmes de leur territoire (Muller, 1992). Les résultats publiés par les deux organismes de recherche leur permettent d'affirmer qu'il est non seulement juste d'un point de vue éthique, mais économiquement justifiable, d'améliorer les conditions de déplacement des personnes handicapées, puisque les bénéficiaires de cette action publique sont en fait très nombreux.

Pourtant l'examen des projets locaux de tramway a révélé que l'accessibilité aux personnes à mobilité réduite est curieusement concentrée sur celle des personnes handicapées, notamment les plus visibles, les personnes en fauteuil roulant et les aveugles. Ainsi, autant la notion de personnes à mobilité réduite a un grand pouvoir de persuasion pour justifier une action publique, autant cette catégorie de PMR ne semble pas très opératoire : les besoins des parents avec des poussettes d'enfant ou les usagers de transport avec des bagages lourds ne sont pas intégrés lorsque les dispositifs techniques sont développés - les différents acteurs préférant la typologie des types de déficience pour travailler concrètement sur les solutions d'accessibilité. Ils adoptent le postulat selon lequel si l'accessibilité est possible pour les lourds handicaps, elle l'est également pour les autres (CERTU, 2006). Ce principe n'a pas fait l'objet d'une vérification scientifique portant sur les capacités et besoins fonctionnels de chacune des différentes populations intégrées dans la catégorie Personnes à mobilité réduite. La justification d'un tel postulat est venue, a posteriori, d'un effet, non anticipé, sur la vitesse commerciale du tramway accessible grenoblois, remarquée également lors de l'introduction des bus à plancher surbaissé. Cependant ce constat de vitesse supérieure à celle attendue ne permet pas de prouver que chaque usager a vu ses conditions d'accès améliorées mais seulement que l'ensemble des montées/descentes de tous les clients se réalise en moins de temps - ce qui n'exclut pas des problèmes d'accessibilité pour certaines personnes.

Ainsi il apparaît que l'usage de la catégorie de PMR est davantage un instrument de légitimation de l'action publique accessibilité qu'un collectif auquel s'identifie tous ses membres. Alors que Myriam Winance précisait que dire c'est identifier et rassembler un collectif, positionner et habiliter (Winance, 2003) en présentant les différents modèles du handicap, l'étude menée ici révèle que les différents groupes de pression sont toujours organisés par type de déficience : autant les associations du handicap moteur expliquent que les personnes avec des bagages ou des poussettes vont bénéficier des améliorations réalisées pour les personnes en fauteuil roulant, autant l'apparition de l'expression Personnes à mobilité réduite n'a pas bouleversé le champ des associations de personnes handicapées en le remodelant autour de plusieurs situations de handicap. 


\section{Conclusion}

Le tramway moderne est devenu le moyen de transport de surface le plus accessible aux personnes à mobilité réduite — résultat symptomatique d'une lente maturation séquentielle des préoccupations sociétales. Ainsi les premières solutions ont cherché à satisfaire les revendications des associations qui se sont mobilisées dès l'origine, à savoir celles des personnes se déplaçant en fauteuil roulant. Les objectifs accéder, monter et descendre ont été atteints dès les années 1980. Le problème de l'information (visuelle et sonore) a mis plus de temps à s'inscrire à l'agenda institutionnel des autorités organisatrices des transports. Le caractère sécuritaire des dispositifs n'a pas été explicitement exposé dans cet article, mais il constitue une préoccupation constante de tous les acteurs locaux. L'autonomie a été une demande très forte exprimée dès le projet grenoblois. La non-stigmatisation, c'est-à-dire l'accès dans des conditions similaires aux autres usagers, est apparue beaucoup plus récemment : la personne handicapée ne doit pas avoir à se déclarer comme telle, même vis-àvis d'un agent du service public de transport, en l'occurrence le conducteur du tramway. Enfin, il est à noter que l'intégration des besoins des personnes handicapées psychiques et cognitives demeure le dernier chantier pour répondre complètement aux objectifs de la loi n²005-102 du 11 février 2005 pour l'égalité des droits et des chances, la participation et la citoyenneté des personnes handicapées. Notre analyse a montré que la prise en compte des besoins des personnes en fauteuil roulant et une manifestation de leurs associations au niveau local avaient été concomitantes - révélant l'impact de cette mobilisation. Il est toutefois frappant de constater que l'absence de dispositifs d'accessibilité pour les personnes handicapées cognitives et psychiques est doublée d'une absence de leurs associations représentatives dans les processus décisionnels d'implantation de tramway, en raison d'une très faible mobilisation de ces associations sur la question de l'accessibilité et des besoins des personnes handicapées mentales mal identifiés par les acteurs locaux. Pourtant plusieurs études ont cherché à connaître les besoins spécifiques de ces personnes dès 1986 et elles ont suggéré des solutions. Il serait intéressant de comprendre de quelle manière les résultats de ces études ont été diffusés dans le champ des professionnels des transports et des associations locales.

L'étude de différents projets d'implantation de tramway a permis de mettre en lumière l'évolution temporelle qu'a connue la notion d'accessibilité. Elle montre de manière frappante le rôle crucial tenu par les acteurs dans cette marche inexorable vers un tramway accessible et la nécessité d'une mobilisation des énergies locales. Le développement d'une expertise sur l'accessibilité est venu conforter cette tendance, en faisant passer les souhaits des personnes handicapées du vœu pieu à la solution concrète et accroissant ainsi les tensions entre ce qui est, ce qui pourrait être et ce qui devrait être (Padioleau, 1982). Des pressions internationales se sont également exprimées, diffusant une nouvelle vision du handicap et légitimant un certain type d'action en faveur des personnes handicapées. Enfin si la catégorie de personnes à mobilité réduite n'est pas très opératoire pour le développement des solutions d'accessibilité et peut être qualifiée d'incantatoire, il n'en est pas moins vrai que la mise en accessibilité du tramway facilite le déplacement de nombreuses personnes et qu'elle a des avantages en termes d'exploitation.

La présente analyse a montré que l'innovation de l'accessibilité aux personnes handicapées a principalement été impulsée au niveau local et qu'elle est le résultat de compromis entre les contraintes et les enjeux des autorités organisatrices des transports, aidées de leur maître d'œuvre, de leur exploitant, des associations de personnes handicapées et des constructeurs de 
tramway. L'approche par le bas semble donc ici avoir un facteur explicatif supérieur à l'approche par le haut. Toutefois, si nous nous étions uniquement focalisés sur ce premier type d'approche, le rôle de l'État aurait pu nous échapper. Or, si l'État central n'a pas imposé des normes de construction comme cela était prévu par la loi de 1975, il a accompagné tout le processus d'évolution de l'accessibilité. Par sa présence, il a d'abord légitimé l'action des acteurs locaux visant l'accès au tramway des personnes en fauteuil roulant en finançant pour partie la recherche et développement et en lui donnant, sinon légalement, du moins pratiquement, le caractère d'intérêt général. Puis il a participé à la diffusion de cette innovation en ne subventionnant plus que les tramways accessibles. Enfin, il a récemment concrétisé le changement de la signification de l'accessibilité en lui donnant une valeur juridique. Ses services déconcentrés participent également à ce processus en adoptant une posture de contrôle, permise par leurs missions. Ainsi, les résultats obtenus nous incitent à encourager, si l'objectif d'une recherche est la mise en lumière de facteurs explicatifs, l'adoption d'une démarche heuristique multipliant les approches. 


\section{Abridged version}

\section{Introduction}

Accessibility for people with reduced mobility is one of the principal improvements to have marked urban transportation in the last twenty years. The tram is the ideal transport system for observing this change. This paper sets out to clarify the concept of accessibility for people with reduced mobility and examine how this concept has changed over time. It also aims to identify the factors which have encouraged this change. In this context, the paper examines the explanatory capability of two processes: a top-down process and a bottom-up process. To investigate these issues, several tram projects (Grenoble, Strasbourg and Lyon) have been studied in order to reveal the factors which have encouraged locally the emergence of one technological solution or another.

\section{Legal instruments, between a symbolic constraint and an institutional framework}

\subsection{A very recent legal definition}

France has recently reformed its policy in relation to people with disabilities: the act of 11 February 2005 makes it necessary for all public transport services to be made accessible before 2015. To put it more clearly, the entire transport chain, which includes the built environment, the road, public spaces, transport and intermodal connections must be fully accessible for people with disabilities or reduced mobility. This law defines a concept of disability as something which limits activities or restricts participation in the life of society. The concept of people with reduced mobility has been defined since 2001 by a European directive: it includes not only people with disabilities, but also people of small stature, the elderly, pregnant women, and people travelling with luggage, shopping trolleys or children. A decree followed which defined what is meant by an accessible public transport vehicle. This must be designed to permit the person with a handicap or reduced mobility to board, alight, use all the on-board services, identify their position, find their direction and obtain the information required to make their journey under all circumstances - under conditions which are equal to those of other users with the greatest possible autonomy and without danger.

\subsection{Previous legal regulation of a different type: was accessibility a policy goal or a policy means?}

Those involved in transportation have only recently been provided with a very clear definition of what is an accessible vehicle. Nevertheless, the ability of people with disabilities to travel is a public problem that has for some time featured on the French government's institutional agenda. Thus, the act of 30 June 1975 already recommended the provision of access to institutions which are open to the general public whenever the aptitude of the disabled people and their environment allowed. Measures have been taken with regard to the subject on two occasions in the sphere of transportation. A decree specifying how the 1975 act should be enforced required local and regional authorities to draw up a programme to upgrade transport services and installations. These programmes were intended to determine the measures to be implemented in order to improve the accessibility to scheduled services insofar as the type of services and the operating constraints were not obviously incompatible with the very nature of the impairment, or place at the disposal of the people with disabilities a transport system 
which meets their needs if they were. The 1975 act did not define the concept of people with disabilities. Local actors were responsible for deciding who should benefit from accessibility. An examination of official French government thinking shows that the two technical solutions corresponded to two different groups: accessibility to scheduled services for autonomous individuals with slight impairment and the creation of specialized services for non autonomous individuals with severe impairments, of whom the archetype was wheelchair users.

\section{A tramway project, a configuration of actors, accessibility solutions}

Between 1975 and 2005, national policy thus fixed an objective - improving the mobility of disabled people - but did not give priority to any approach. This legal context created a normative environment which has influenced the behaviour of local actors, particularly those in charge of a tramway project. However, since their introduction in France, the level of accessibility of trams has been constantly increasing. The rest of this paper will seek to show that the solutions that have been adopted depend to a large extent on the local actors who are involved in the projects, the constraints facing them, their concerns and their view of accessibility. Particular attention has been given to three projects which are characteristic of three different levels of accessibility.

\subsection{Grenoble, a pioneering city}

The problem of accessibility for people with disabilities was already on the agenda before 1975. For some years prior to this, the city of Grenoble had received a large number of state subsidies to modify its roads and housing. A specialized transport service for wheelchair users and the visually impaired was set up in 1979. When the tramway project entered the decisionmaking phase, associations representing people with disabilities, in particular wheelchair users, refused the standard French tram which at the time had a high floor and several steps. In response to this demand, the local officials contacted the Ministry of Transport. The French standard tramway technical committee, which brought together representatives of the State, local authorities, public transport operators, project managers and centres of expertise examined the question. After a lengthy exchange of ideas between engineers, it was decided that it was technically possible to lower the vehicle floor. Significant State investment was necessary in order to initiate a new phase of research and development for a low-floor tram. A study demonstrated that a ramp was necessary to provide access for wheelchair users. Such a device was developed and fitted to two of the doors of each tramcar. Thus, although at national level, wheelchair users were perceived as not being legitimate beneficiaries of accessibility, they were the first to join forces to demand - and obtain - access to a tramway.

\subsection{The Strasbourg tramway project, importing good practice}

The Strasbourg tramway was brought into service in 1994, it has a low floor over its entire length. This solution is not the outcome of a demand on the part of associations representing the disabled - the solution adopted in Grenoble was generally speaking satisfactory - but on the part of local officials, who felt that the Grenoble tramway was outdated: they wanted an entirely low floor vehicle to facilitate transfers and improve the integration of the tramway in the urban environment. 
With regard to accessibility, the Strasbourg tram differed from the others by its provision of next station announcements and visual displays. In addition, the destination and the waiting time of the next tram are displayed at the stop. These technological developments are the concrete outcome of the inclusion of people with visual or hearing impairments among the beneficiaries of accessibility. They are the outcome of the trust between technicians and associations of disabled persons that had been set up during the drafting of a charter entitled Ville et handicap (the City and Disability).

\subsection{In Lyon, the Grenoble solution brought into question}

While in the 1980s, safe and autonomous access to the vehicle was the main problem, now an absence of discrimination is demanded. The Grenoble ramps, which were also used on the Strasbourg tram, are currently criticized because of the need for users to announce their impairment. This is because the ramp is not deployed automatically each time the doors are opened: the driver needs to recognize the person on the platform to bring it into play. In order to overcome this problem, the Lyon tram is fitted with an automatic ramp to bridge the gap between the vehicle and the platform which moves into place each time the doors are opened. The development of this technology is the result of a great deal of work on the part of the accessibility advisor to the Mayor of Lyon who, himself being disabled, supported a genuine policy for accessibility. The accessibility advisor, working at the interface between political and associative networks, persuaded the company building the tramway to develop automatic ramps, while the originally proposed project merely complied with the recommendations of the liaison committee for transport accessibility, a multipartnership committee supervised by the Ministry of Transport.

The needs of persons with visual and hearing impairments were not forgotten, as all the tramcars are equipped with a system of next station voice announcements and visual displays; tactile strips have been installed along platforms and audible traffic signals have been installed in the vicinity of the tramway. The only factor which prevents the Lyon tramway from fully meeting the requirements of the Act of 11 February 2005 is that the project does not take account of the needs of people with mental disabilities. While a considerable amount of research has highlighted their needs, they have not been clearly identified by the local actors. Furthermore, the local associations who represent this type of disability do not consider themselves directly concerned by the problem of accessibility and have not made any demands in this area.

\section{Accessibility, an innovation at the intersection of individual and structural dynamics}

These tram projects show that the level of accessibility has been constantly improving: the dynamic is marked by processes of knowledge accumulation and the reformulation of the meaning of the concept of accessibility. This change can be explained by four factors:

- joint work on the part of five principal actors,

- the development of technical expertise on accessibility,

- stances adopted at international level,

- the creation of the category of people with reduced mobility. 


\subsection{An essential quintet}

In spite of their differing constraints and concerns, five principal actors have worked on each of the projects that we will examine in order to obtain a tramway that is increasingly accessible:

- the transport authorities, which have mobilized the energy and expertise and play a role with regard to legitimizing and filtering the demands from associations;

- the project manager which provides the link between the demands of the local authorities and the constraints of the tram manufacturer;

- the tram manufacturer, which has scarce expert knowledge, but which is also constrained by its industrial strategy and internal organization;

- associations of disabled people, who have sole responsibility for defining the needs of people with disabilities and who consequently express their demands;

- the State, with its large number of instruments, acts, regulations, technical standards, subsidies and controls.

\subsection{The development of technical expertise}

The changes of the concept of accessibility are also explained by the development of technical expertise in this area. Many dynamics have played a part in this: movement of engineers within the various transport authorities or their operators, the involvement of several actors in different projects, a dual process of acculturation and awareness raising through government working groups and technological monitoring performed by the tram manufacturers and the project managers.

\subsection{Accessibility, an international demand}

The stances of international and European bodies have also supported demands for accessible public transport. The United Nations, the European Conference of Transport Ministers and the European Union all encourage their member countries to make public transport accessible to people with disabilities.

These stances go hand in hand with a change in the international definition of disability. In the early 1980s, the World Health Organization (WHO) took what is known as the medical view of disability: disability is the outcome of a difference between the functional capacities of a disabled person and the average capacities of healthy individuals. This view was criticized as excessive and the WHO recently adopted the international functional classification which combines the medical and social models of disability. This looks at the issue the other way round. A person is only disabled in a given situation: our attention should be turned away from the individual and focused on the disabling situations. This reversal in the international representation of disability legitimizes measures that encourage accessibility.

\subsection{People with reduced mobility, a strongly evocative category, but one which has not yet had a practical impact}


A final factor legitimizes the provision of accessibility: the use of the category of people with reduced mobility (PRM) to extend the number of beneficiaries of accessibility and thus justify the budgets allocated to measures which no longer target only wheelchair users but all those with reduced mobility. However, in the different tramway projects we have considered the local actors did not use this category hen developing technical solutions: they prefer to remain with an approach based on the type of disability. Because accessibility improves comfort for everyone, the concept of PRM is extremely evocative, but it obviously has yet not had a practical impact.

\section{Conclusion}

The tramway is currently the transport mode which is the most accessible for disabled people, as the result of a slow and gradual maturing of societal concerns. The new accessibility requirements laid down by the act of 11 February 2005 and its application decree, the objectives of gaining access, boarding and alighting were achieved in the mid-1980s. The need to be able to identify one's location and access information has been addressed since the Strasbourg project. The demand for non-stigmatization is one which appeared more recently. It should be noted that the integration of people with mental and cognitive disabilities is the last stage which is necessary to meet fully the objectives of the 11 February 2005 Act. It is striking to observe that the technical solutions that provide accessibility have developed in parallel with the involvement of the associations that represent the different types of disability: the associations of wheelchair users were the first to take action and this was the first problem to be tackled. The associations that represent people with cognitive disabilities have not yet become involved in the field and their needs are still ignored.

The study of different tramway projects has highlighted the changes that the concept of accessibility has undergone. It demonstrates in a striking manner the crucial role played by local actors in this inexorable progress. Public action with regard to accessibility for people with disabilities has thus principally been driven at local level and has been the outcome of compromises with regard to the constraints and concerns of the different actors. This finding gives the impression that a bottom-up process is in this case a better explanatory factor than a top-down process. However, the different stances adopted by central government and their local services which the study has identified would no doubt not have been highlighted if we had focussed solely on the first process. This would encourage us to adopt a heuristic procedure that employs many approaches in order to identify all the explanatory factors. 


\section{Références}

Artaud-Macari, J., Bachelier, C., 1977. Les transports au service des handicapés. Rapport du groupe de travail interministériel sur les problèmes posés par le transport. Secrétariat d'État aux Transports, Paris.

CERTU, 2006. Schémas directeurs d'accessibilité de transports collectifs urbains, Analyse de cas. Rapport CERTU, Lyon, France.

Chapireau, F., 1988a. Le handicap impossible. Analyse de la notion de handicap dans la loi d'orientation du 30 juin 1975 ( $1^{\text {re }}$ partie). Annales médico-psychologiques, 146 (7), 609-631.

Chapireau, F., 1988b. Le handicap impossible. Analyse de la notion de handicap dans la loi d'orientation du 30 juin 1975 ( $2^{\mathrm{e}}$ partie). Annales médico-psychologiques, 146 (8), 691-706.

Collomb d'Eyrames, O., 2002. Eléments de réflexion sur le processus d'accessibilité des personnes handicapées à Grenoble. Université Pierre Mendès France Grenoble II, France.

Crozier, M., Friedberg, E., 1977. L'acteur et le système. Les contraintes de l'action collective. Le Seuil, Paris.

Florès, J.-L., 1984. Accessibilité des transports et de la ville aux personnes handicapées. Rapport du groupe de travail Recherche et Technologie de la Mission Fraysse-Cazalis. Institut de Recherche des Transports, Bron, France.

Florès, J.-L., Blanchet, V., Germain, C., 1984. Evaluation des solutions techniques susceptibles de rendre le tramway standard accessible aux utilisateurs de fauteuil roulant. Institut de Recherche des Transports, Centre d'Evaluation et de Recherche des Nuisances et de l'Energie, Bron, France.

Hauet, É., Ravaud, J.-F., 2002. Handicap, gênes ou difficultés ressentis par les personnes dans le cadre de leurs déplacements en dehors du domicile. CNRS, Villejuif, France.

Hermelin, M., Leloup-Lambard, A., Stevoux, P., Thery, C., Velche, D., 1986. Les personnes handicapées mentales et les transports en commun. Dialogues Snadoc, Paris.

Heyrman, É., 2002. Les personnes à mobilité réduite : vers une mise en accessibilité globale des projets d'aménagement publics? L'exemple lyonnais. Mémoire de Travail de Fin d'Etudes, Ecole Nationale des Travaux Publics de l'État.

Heyrman, É., 2003. Entre normes centrales et contextes locaux : la mise en accessibilité des systèmes de transports. Les exemples des tramways et métro lyonnais et grenoblois. Mémoire du DEA Politiques Publiques et Gouvernements Comparés, Université Lumière Lyon II, France.

IAURIF, 1997. Les déplacements des personnes à mobilité réduite et/ou en situation de handicap en Île-de-France. Rapport de synthèse IAURIF.

Latour, B., 1992. Aramis ou l'amour des techniques. La Découverte, Paris. 
Menétrieux, L., 2005a. La mise en accessibilité du tramway aux personnes à mobilité réduite : depuis Grenoble, quelles évolutions et quels acteurs? Mémoire de Travail de Fin d'Etudes, Ecole Nationale des Travaux Publics de l'État.

Menétrieux, L., 2005b. Entre accessibilité aux personnes à mobilité réduite et lutte contre le tout-automobile en ville : le tramway français, un révélateur des ambitions de la société contemporaine. Mémoire du Master Transports, Espace, Réseaux, Université Lumière Lyon II, France.

Meny, Y., Thoenig, J.-C., 1989. Politiques Publiques. PUF, Paris.

Muller, P., 1992. Entre le local et l'Europe. La crise du modèle français des politiques publiques. Revue Française de Science Politique, 42 (2), 275-297.

Padioleau, J.-G., 1982. L'État au concret. PUF, Paris.

Ratel, L., 1996. Hubert Dubebout le bâtisseur 1965-1983. Éditions Belledone.

Ravaud, J.-F., 1999. Modèle individuel, modèle médical, modèle social : la question du sujet. Handicap, revue des sciences humaines et sociales, 81, 64-75.

Sanchez, J., 1997. Enjeux concrets et symboliques de l'accessibilité, In: Ravaud, J.-F., Didier, J.-P., Aussilloux, C., Aymé, S. (eds.), De la déficience à la réinsertion, Recherches sur les handicaps et les personnes handicapées. INSERM, Paris, 139-146.

Sanchez, J., Velche, D., 1999. Mobilités réduites : les épreuves de l'accessibilité. In: Joseph, I. (ed.), Villes en gares. L'Aube, Paris, 272-291.

Soulas, C., 2003. Les transports guidés sur pneus dits "systèmes intermédiaires" dans le contexte de l'innovation en transport collectif urbain. Transports Urbains, 105, 3-11.

Winance, M., 2003. Pourriez-vous être politiquement correct lorsque vous parlez des personnes handicapées ? Sur la force du discours dans le champ du handicap. Handicap, revue des sciences humaines et sociales, 97. 